
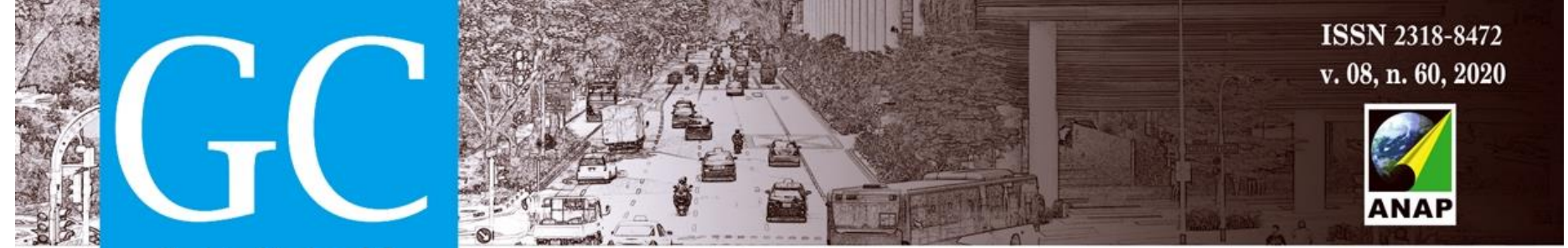

Revista Nacional de

Gerenciamento de Cidades

\title{
Destinos e Distâncias da Caminhada Utilitária: um estudo na cidade de Rolândia - PR
}

Destinations and distances of utilitarian walking: a study in the town of Rolândia-PR

Destinos y distancias de la caminata utilitaria: un estudio en la ciudad de Rolândia-PR

\author{
Mariana Gimenez Casarim \\ Mestranda, UEL, Brasil. \\ marianagcasarim@hotmail.com
}

Luís Gustavo Rocha Pereira

Graduando, UEL, Brasil.

luisgrpereira@uel.br

Ana Luiza Favarão Leão

Doutoranda, UEL, Brasil.

analuiza.favarao@uel.br

Milena Kanashiro

Docente, UEL, Brasil. milena@uel.br 


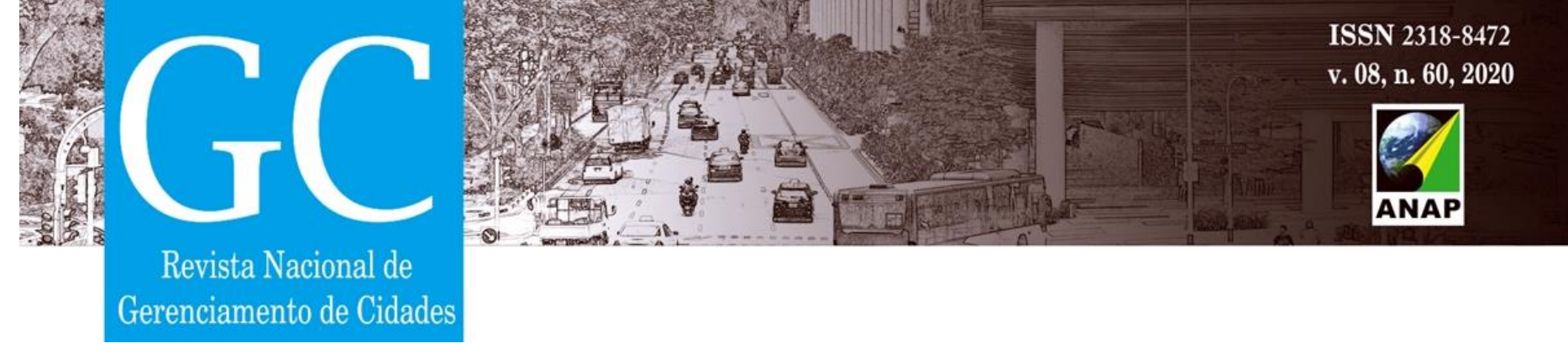

\section{INTRODUÇÃO}

Pesquisas têm mostrado de modo recorrente que o risco de desenvolver DCNTs (doenças crônicas não transmissíveis) diminui com a prática de atividades físicas (CHODZKO-ZAJKO et al., 2009). A caminhada é a forma mais frequente de atividade física entre adultos (RAFFERTY et al., 2002) e, quando inserida no cotidiano, promove benefícios à saúde (PUCHER; BUEHLER, 2010). O deslocamento a pé permite, além disso, a diminuição do transporte motorizado e, consequentemente provoca a diminuição dos congestionamentos no trânsito e uma melhor qualidade do ar (MOUDON; LEE, 2003).

A caminhada pode ser analisada enquanto comportamento ativo influenciado pelo ambiente (LESLIE; CERIN, 2008), de modo que existam áreas mais "caminháveis" que outras. Southworth (2005) define o conceito de caminhabilidade como a característica do ambiente construído que apoia e encoraja a caminhada. Neste sentido, índices de caminhabilidade tem sido aplicado em várias cidades do mundo (TSIOMPRAS; PHOTIS, 2017; HABIBIAN; HOSSEINZADEH, 2018) e tem como um dos componentes o uso misto do solo. Parte-se do pressuposto, que áreas com uma variedade de usos seriam mais caminháveis. $O$ índice mais disseminado tem sido aquele desenvolvido por Frank et al. (2010) o qual define cinco categorias de uso para avaliação: 1. Residencial; 2. Comercial; 3. Serviço; 4. Institucional; 5. Entretenimento. No entanto, tais categorizações se tornam generalistas e, estudos tem apontado especificidades de destinos, como a separação dos tipos de compras (NATHAN et al., 2012) e os tipos de instituições de educação (TSIOMPRAS; PHOTIS, 2017), os quais são potenciais influências no comportamento de caminhada.

O deslocamento a pé pode ser reconhecido em dois comportamentos ativos diferentes: a caminhada utilitária e a caminhada recreativa. Segundo Kang et al., (2017), caminhada utilitária é aquela que possui como fim um destino específico, já a recreativa está associada à prática de atividade física, sem relação a destinos. Essa distinção é relevante, pois devem ser adotadas estratégias específicas para compreender as diferenças ente os tipos de caminhada (KANG et al., 2017). A caminhada utilitária vem sendo apontada como o elemento central no apoio à vida sustentável nas cidades e pode promover níveis recomendados de atividade física, se inserida na rotina diária (DOESCHER et al., 2014). Evidências da literatura têm associado, de modo consistente, a caminhada utilitária à disponibilidade e proximidade de destinos relevantes (SUGIYAMA et al., 2012) e, esta relação pode variar conforme o tipo de destino (KING et al., 2015).

Portanto, a especificação de destinos tem sido abordada em estudos recentes como instrumento de compreensão espacial e no entendimento de quais destinos podem favorecer ou dificultar a opção pelo caminhar (MILLWARD; SPINNEY; SCOTT, 2013; HABIBIAN; HOSSEINZADEH, 2018); e na diversidade e frequências de destinos, a partir de associações entre diferentes grupos sociodemográficos (CERIN et al., 2007). Soma-se à essa discussão - a relação do deslocamento a pé com o ambiente construído - ainda foi pouco investigada em países de economias em desenvolvimento. A urbanização da América Latina se deu de maneira 


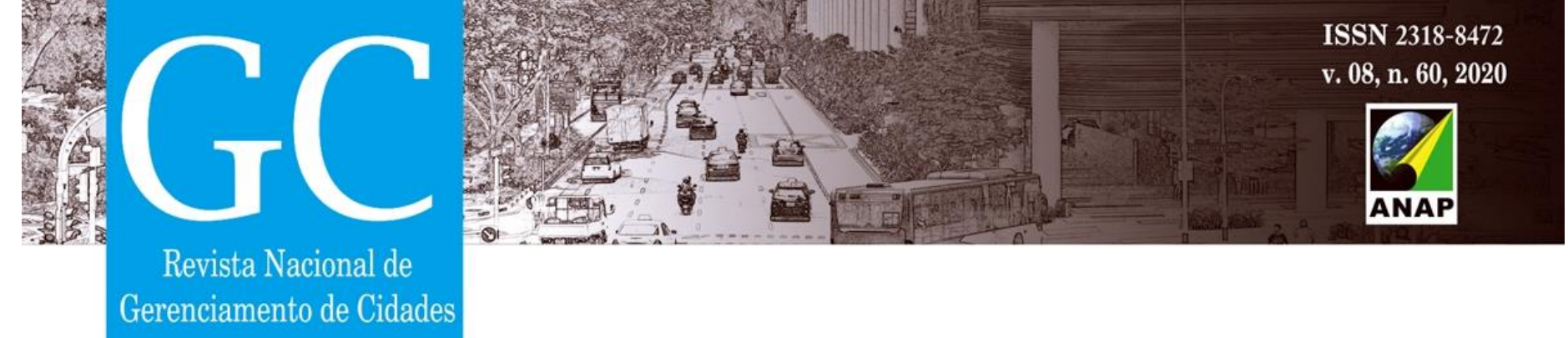

diferenciada dos países desenvolvidos (REIS et al., 2013) e, os estudos existentes no Brasil são direcionados quase que exclusivamente para grandes cidades, cujas características culturais e ambientais diferem das cidades menores (NAKAMURA et al., 2013).

Assim, pesquisas de caminhabilidade devem ser encorajadas para compreensão dos atributos comportamentais e ambientais, em diferentes cidades (SUGIYAMA et al., 2012). Dessa maneira, os resultados da pesquisa, na compreensão do deslocamento ativo, poderão fornecer subsídios para diretrizes de organização de cidades, bem como para subsidiar implantação de políticas públicas para a melhoria da qualidade de vida da população na busca de cidades mais saudáveis e sustentáveis.

\section{OBJETIVOS}

Deste modo, fica evidente a lacuna de pesquisa sobre a caminhabilidade em cidades pequenas e médias brasileiras, sobretudo na abordagem dos destinos como foco da análise. 0 objetivo desta pesquisa é analisar a relação dos destinos e das distâncias da caminhada utilitária em Rolândia-PR. Para alcançar tal objetivo definem-se três objetivos específicos: o primeiro, elaborar e aplicar uma categorização de destinos, baseada em referências na literatura e, segundo analisar a espacialização dos pontos de destino por subcategoria e suas respectivas distâncias e por fim, uma aproximação das variáveis sociodemográficas.

\section{METODOLOGIA}

A estratégia de pesquisa adotada foi o estudo de caso (YIN, 2001), tendo em vista a natureza complexa e contemporânea do objeto de análise. A cidade de Rolândia, no norte do Paraná, foi escolhida como estudo empírico, dada a disponibilidade de dados e sua representatividade populacional enquanto município médio brasileiro. Rolândia tem área de $459,024 \mathrm{~km}^{2} \mathrm{e}$ população estimada de 66.580 habitantes (IBGE, 2020). O banco de dados foi fornecido para o Grupo de pesquisa, pelo ITEDES, e corresponde à pesquisa Origem-Destino (OD) do Plano de Mobilidade de Rolândia - a pesquisa conta com dados auto reportados, incluindo variáveis sociodemográficas, características de origem e destino das viagens. Dos dados referentes aos deslocamentos a pé, um total de 391 viagens foram georreferenciados em SIG (Sistema de Informação Geográfica).

A pesquisa OD coleta informações relativas à viagem, incluindo a classificação dos destinos por motivos (1. Trabalho/indústria; 2. Trabalho/comércio; 3. Trabalho/serviço; 4. Escola/educação; 5. Compras; 6. Médico/dentista/saúde; 7. Recreação/visitas; 8. Residência; 9. Outros). Observando-se a categoria de motivo proposta pela pesquisa OD, os classificados como "8. Residência" não foram considerados $(n=225)$. Foram excluídos também destinos com informações ausentes e sem a definição do uso específico $(n=30)$. Pela natureza dos dados auto reportados do questionário, embora havia pontos de destino com informações incompletas, estes foram inferidos a partir de dois critérios: 1. na associação entre motivo e uso específico do lote; 2. na associação do motivo e parte de vias com o uso correspondente, utilizou-se o 


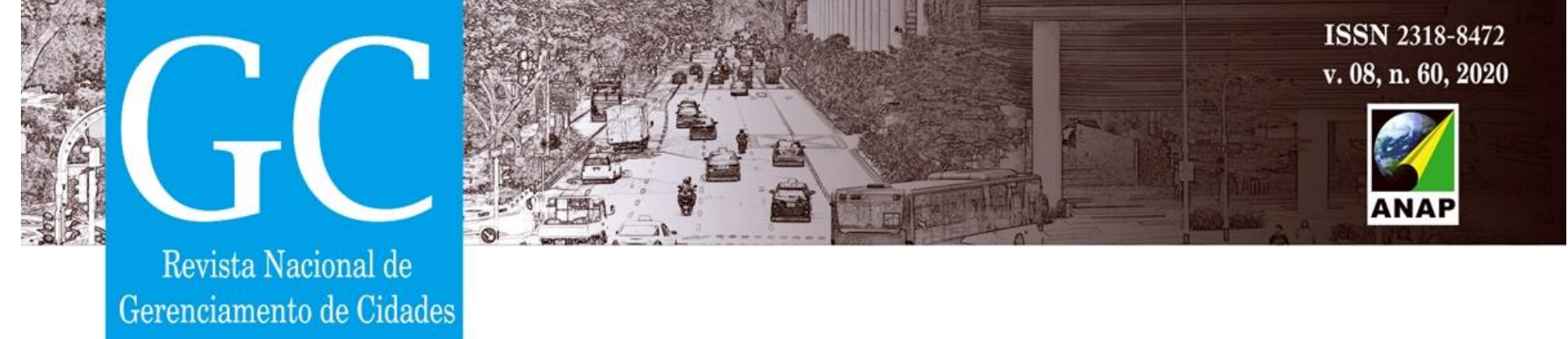

centroide da via. Ainda, para dados mais precisos utilizou-se os recursos do Google Maps e Google Streetview. O banco de dados final resultou em 166 destinos georreferenciados e seus respectivos usos e atividades específicas.

As viagens registradas foram espacializadas em procedimentos que conectam origens e destino geocodificados, na definição de rotas georreferenciadas. No uso da caixa de ferramentas Geocoding Tools e o recurso Address Geocode do ArcGIS 10.6, as 166 rotas foram geocodificadas. Posteriormente, a partir das ferramentas do Spatial Analyst do ArcGIS Online e Connect Origins to Destinations, para espacialização das rotas. Esta ferramenta mede a distância geodésica entre pares de pontos seguindo a rede de ruas que permitem o trânsito de pedestres, considerando o caminho mais curto possível. Tais rotas foram sobrepostas, resultando no mapa de carregamento, pelo qual é possível identificar quais trechos de via tiveram mais viagens. Somase o uso de representação de superfície de densidade do procedimento estatístico-espacial Densidade de Kernel, para indicar agrupamentos por destinos.

\section{RESULTADOS E DISCUSSÕES}

\section{CATEGORIAS}

A partir da categoria de 9 motivos referida anteriormente, buscou-se criar outra, mais específica (Tabela 1). Pela revisão bibliográfica de pesquisas existentes, verificou-se que as categorias "Trabalho" e "Educação" aparecem frequentemente em estudos sobre destinos dada a relação destas à intensidade de deslocamentos diários regulares e constantes (CHATMAN, 2003; CERIN, 2007).

O motivo "Trabalho" dividido em três classes foi mantido. Por outro lado, a categoria "Educação" apresenta diferentes graus de ensino, que estão relacionados à idade e comportamento dos pedestres. Estudantes de CMEI e Escola Municipal são crianças menores, que necessitam de acompanhantes: a idade indicada para uma criança se deslocar de forma independente para fins educacionais está comumente entre 10 e 16 anos (MOURA; CAMBRA; GONÇALVES, 2017). 
Tabela 1 - Organização das categorias e subcategorias

\begin{tabular}{|c|c|c|c|c|c|}
\hline & 1. Trabalho & 2. Educação & 3. Compras & 4. Saúde & 5. Recreação \\
\hline Subcategoria & $\begin{array}{l}\text { 1.1 Trabalho } \\
\text { na indústria }\end{array}$ & $\begin{array}{l}2.1 \text { Educação não } \\
\text { especificado* }\end{array}$ & $\begin{array}{l}\text { 3.1 Comércio } \\
\text { geral }\end{array}$ & $\begin{array}{l}4.1 \text { Consultórios e } \\
\text { farmácias }\end{array}$ & 5.1 Igreja \\
\hline Respondentes n (\%) & $3(1,82 \%)$ & $8(4,85 \%)$ & $5(3,03 \%)$ & $\begin{array}{l}4(2,42 \%) \\
4.2 \text { Unidades de }\end{array}$ & $4(2,42 \%)$ \\
\hline Subcategoria & $\begin{array}{l}1.2 \text { Trabalho } \\
\text { no comércio }\end{array}$ & 2.2 Colégio Estadual & $\begin{array}{l}3.2 \text { Comércio } \\
\text { de alimentos }\end{array}$ & $\begin{array}{l}\text { Pronto } \\
\text { Atendimento } \\
\text { (UPA) e hospitais }\end{array}$ & 5.2 Visita \\
\hline Respondentes n (\%) & $5(3,03 \%)$ & $40(24,24 \%)$ & $11(6,67 \%)$ & $6(3,64 \%)$ & $9(5,45 \%)$ \\
\hline Subcategoria & $\begin{array}{l}\text { 1.3 Trabalho } \\
\text { em serviços }\end{array}$ & 2.3 Escola Municipal & & $\begin{array}{l}\text { 4.3 Unidades } \\
\text { Básicas de Saúde } \\
\text { (UBS) }\end{array}$ & 5.3 Lazer \\
\hline Respondentes n (\%) & $16(9,69 \%)$ & $41(24,85 \%)$ & & $5(3,03 \%)$ & $4(2,42 \%)$ \\
\hline Subcategoria & & $\begin{array}{l}\text { 2.4 Centro Municipal } \\
\text { de Educação Infantil } \\
\text { (CMEI) }\end{array}$ & & & \\
\hline Respondentes n (\%) & & $4(2,42 \%)$ & & & \\
\hline
\end{tabular}

* abrange destinos possíveis como Colégios Estaduais e instituições particulares

Fonte: os autores, 2020.

A subdivisão da categoria "Compras" foi feita amparada a estudos anteriores que especificam o tipo de comércio (TSIOMPRAS; PHOTIS, 2017; KENYON; PEARCE, 2019), sendo a compra de alimentos uma atividade mais cotidiana. Essa diferença na frequência de uso dos destinos de comércio geral e comércio de alimentos pode ser preditiva de características espaciais distintas. "Saúde" foi subdividida de acordo com o porte das infraestruturas públicas, separando "UPA e hospitais" de "UBS", e pelo caráter privado de "Consultórios e farmácias". (OLIVEIRA et al., 2015). E, por fim, "Recreação" foram divididas de acordo com os comportamentos distintos que seus destinos podem abranger. "Visitas" trata-se de ir a residências de amigos e familiares, sendo estes os únicos destinos de uso residencial considerados no estudo. Desta forma, para uma primeira aproximação de destinos, as categorias são mais gerais e as subcategorias se organizam em 15 subcategorias (Tabela 1 ).

\section{CATEGORIAS E DESTINOS}

A partir da definição de categorias e subcategorias de destino, e a espacialização destes com o carregamento das viagens realizadas a pé para os destinos, possibilita a análise da relação com a configuração espacial da cidade. O desenho original da cidade de Rolândia, datado de 1932, está quase inteiramente inscrito na área delimitada por uma parábola desenhada adjacente à linha férrea - elemento essencial nas cidades fundadas pela Companhia Melhoramentos Norte do Paraná (REGO; MENEGUETTI, 2006). Esse primeiro local de ocupação da cidade constitui o atual centro, e a oeste, no lado oposto da ferrovia, foi executado nos anos 1950 o loteamento da Vila Oliveira (Figura 1). Atualmente, a Vila Oliveira caracteriza-se como um importante subcentro do município. A linha férrea pode ser identificada como um limite entre esses setores: uma barreira, que mesmo penetrável, isola as partes da cidade (LARA; SILVA, 2019), rompe a continuidade do tecido urbano e modifica formas de locomoção intra-urbana (MOUETTE, 1998). 


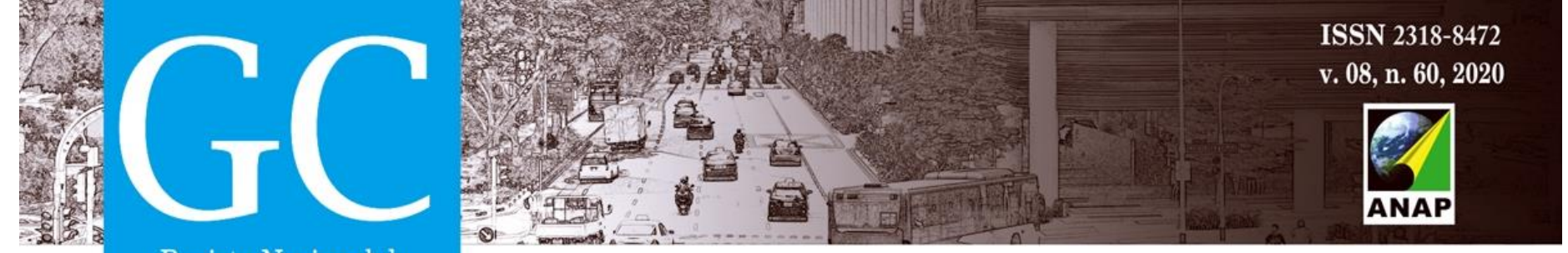

Revista Nacional de Gerenciamento de Cidades

Figura 3 - Destinos de Trabalho

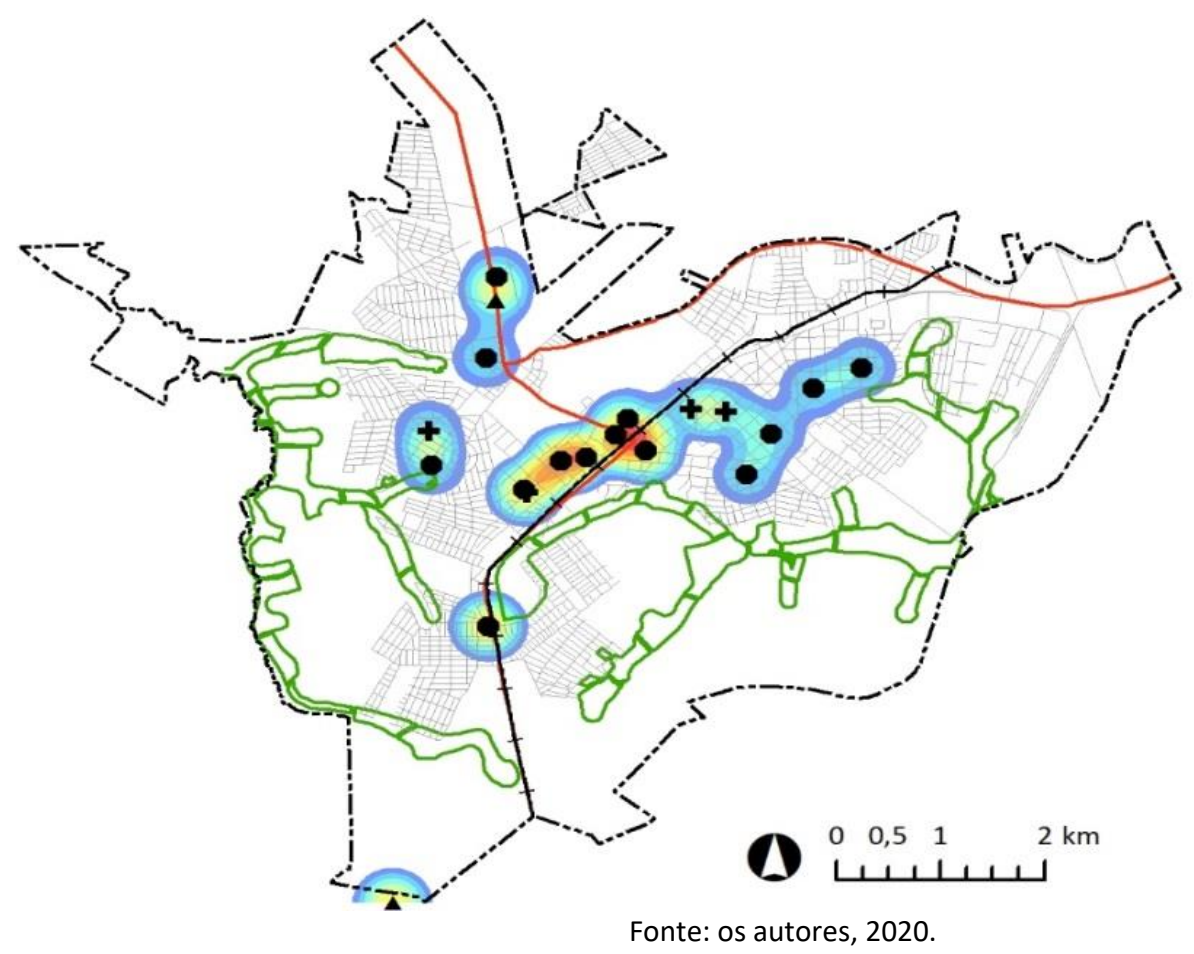

Legenda TRABALHO Subcategoria

A Indústria

+ Comércio

- Serviços

十 Ferrovia

---. Perimetro urbano

- Rodovias

Arruamento

Fundos de vale

Limite de vale

Na sequência, o trabalho em comércio, em menor densidade comparativamente do trabalho em serviços, apresenta uma distância próxima a 1,5 km. Ambas ultrapassam as distâncias caminháveis dos estudos anteriores, porém referem-se às discussões da problemática de morar próximo aos locais de trabalho. Existem duas aglomerações de destinos de trabalho em serviços. A maior densidade de destino está nos arredores da Avenida Presidente Getúlio Vargas - que atravessa a cidade de Rolândia. A região possui facilidade de recebimento e escoamento de produtos para a BR-369. Para os pedestres, esse local é facilmente acessível a partir dos dois lados da linha férrea. Outro local de maior densidade está localizado na área central. A proximidade de ambas as densidades de destinos de trabalho no serviço, nas áreas mais sedimentadas da cidade, define uma distância menor a ser percorrida a pé, cerca de $851 \mathrm{~m}$.

Larsen et al. (2009) apontaram que a distância entre a moradia e a escola é o mais importante fator para determinar se estudante se deslocará em modo não motorizado. Em Rolândia, as viagens realizadas a pé, foram as de maior quantidade para o destino "Educação". As maiores densidades de destinos referem-se aos Colégios Estaduais, geralmente com um maior número de vagas tanto para o Ensino Fundamental II e Médio. As distâncias até esses colégios foram de quase $900 \mathrm{~m}$, e formam duas concentrações, uma em cada núcleo - Vila Oliveira e Centro, a primeira com um volume maior de destino. Deve-se considerar que são crianças acima de 10 anos e adolescentes. As escolas municipais, de Educação Infantil e Ensino Fundamental I, estão mais dispersas na cidade e, localizam-se mais próximas residências a uma distância média de $600 \mathrm{~m}$. Essa distância é similar à dos $\mathrm{CMEl}$, específico para Educação Infantil. Tais resultados indicam um deslocamento proporcionalmente inferior às distâncias caminhadas quando 


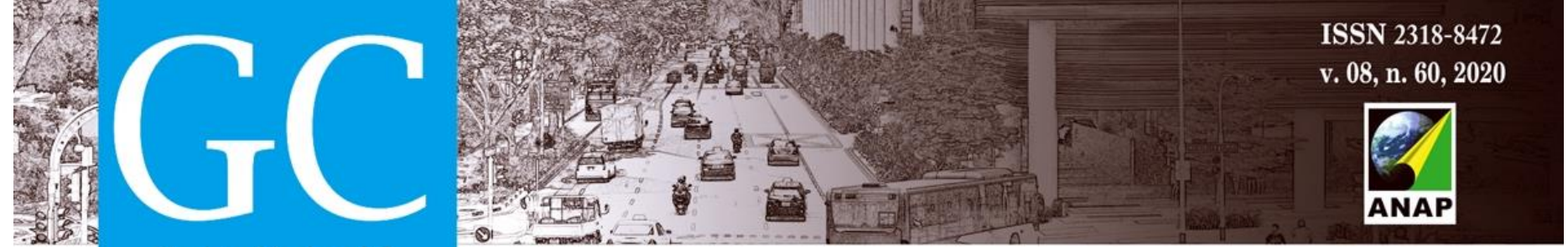

Revista Nacional de

Gerenciamento de Cidades

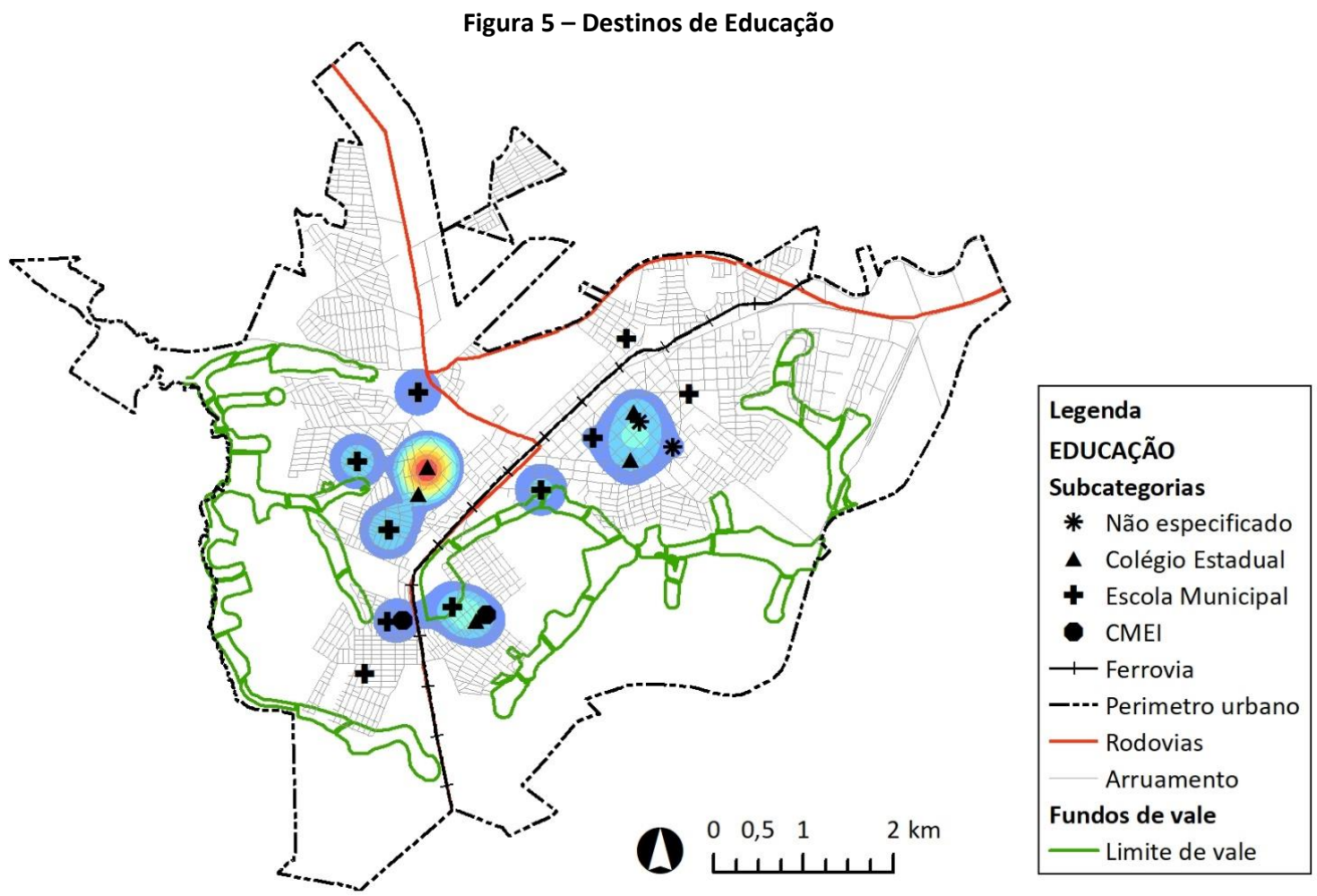

Fonte: os autores, 2020.

Nas subcategorias compras, verifica-se uma expressiva diferença das médias de distâncias de acima de $1 \mathrm{~km}$ para compras em geral e uma proximidade de $779 \mathrm{~m}$ para compras de alimentos (Figura 6). O comércio geral, apesar do baixo número de viagens e dos valores de distância percorrida, apresenta uma concentração de densidade de destinos na área central. São geralmente vinculadas às denominadas compras comparadas, de menor frequência e mais planejamento (KOTLER; ARMSTRONG, 2007). Em contrapartida, distribuído de forma dispersa na área de estudo, o comércio de alimentos contempla a maior parte das viagens relacionadas a categoria com as menores distâncias obtidas. São compras mais frequentes e de localização conveniente para atender a demanda cotidiana. Tais características reforçam comportamentos distintos de pedestres quando analisadas as especificidades dentro da categoria compras para fins de consumos diários - com viagens constantes e menores distâncias relacionados ao ambiente de vizinhança e consumos esporádicos - deslocamentos específicos relacionados a maiores distâncias, identificado por Moura (2010) no contexto de grandes cidades brasileiras. 


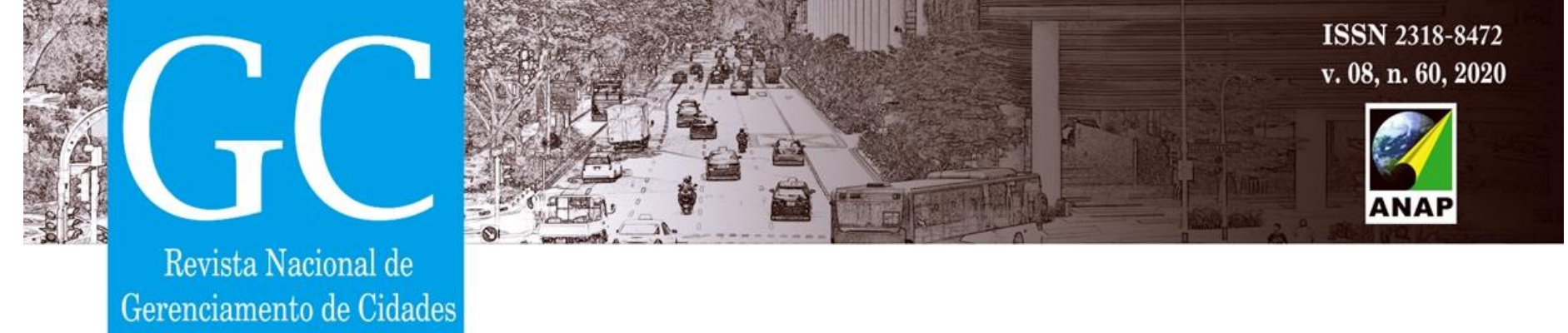

adolescentes, que apontam a condição de renda como fator preditivo de deslocamento ativo para a escola (PABAYO; et al., 2011; HALLAL et al., 2006).

A subcategoria comércio de alimentos apresenta deslocamentos de grande variação de graus de instrução e, a predominância de usuários com idade média mais elevada (Tabela 4). Isso pode estar vinculada a necessidade de aquisição de produtos básicos. Verifica-se nesta categoria como as distâncias das subcategorias de comércio, se relacionam às faixas etárias: o comércio de alimentos, que apresenta menores distâncias, possui prevalência de idosos. A maior participação de idosos em comércio de alimentos, que se encontram dispersos em áreas residenciais, reforça os maiores índices de caminhada desse grupo na vizinhança (LIAO et al., 2020).

Em todas as subcategorias de Saúde, observa-se a prevalência do gênero feminino, um possível impacto da maior preocupação destas com a saúde (IBGE, 2017). A subcategoria com estruturas de maior complexidade, voltada a emergências e tratamento, "UPA e hospitais" apresenta grande concentração de idosos e deslocamentos maiores de moradores com graus mais baixos de escolaridade, alguns dos quais reportaram não ter renda. Opostamente, com estruturas mais simples, a subcategoria "Consultórios e farmácias" é caracterizada por grau mais elevado de instrução e por todos os respondentes possuírem renda (Tabela 4) - isso pode ser um indicativo de maior prevenção entre as classes sociais mais altas e maior necessidade de tratamento entre as mais baixas.

Na categoria Recreação, nota-se que a subcategoria "Visitas" e "Lazer" são marcadas por um perfil com uma idade avançada (Tabela 4), reforçando resultados encontrados em cidades de países desenvolvidos (CERIN et al., 2013; LIAO et al., 2020). Esses valores sugerem que a disponibilidade de destinos de lazer pode ser importante para manter a atividade física na população idosa. Um dos pontos de Lazer significativo é o Centro de Convivência do Idoso. Enquanto nas Igrejas pode-se relacionar a idade adulta e as longas distâncias percorridas. 


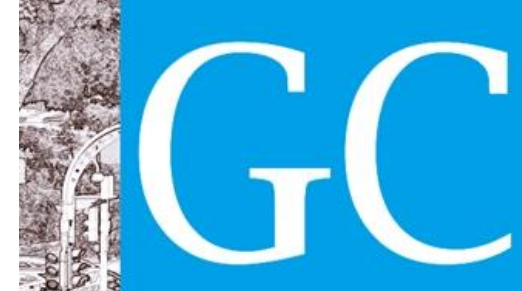

Revista Nacional de

Gerenciamento de Cidades

Tabela 3 - Variáveis sociodemográficas e distâncias por subcategorias (parte 1)

\begin{tabular}{|c|c|c|c|c|c|c|c|c|c|c|c|c|c|c|c|c|c|c|c|c|c|}
\hline & \multicolumn{9}{|c|}{ Trabalho } & \multicolumn{12}{|c|}{ Educação } \\
\hline & \multicolumn{3}{|c|}{ Indústria } & \multicolumn{3}{|c|}{ Comércio } & \multicolumn{3}{|c|}{ Serviços } & \multicolumn{3}{|c|}{ Não especificado } & \multicolumn{3}{|c|}{ Colégio Estadual } & \multicolumn{3}{|c|}{ Escola Municipal } & \multicolumn{3}{|c|}{ CMEI } \\
\hline & $\mathrm{n}$ & $\%$ & $\mathrm{~km}$ & $\mathrm{n}$ & $\%$ & $\mathrm{~km}$ & $n$ & $\%$ & $\mathrm{~km}$ & $\mathrm{n}$ & $\%$ & $\mathrm{~km}$ & $\mathrm{n}$ & $\%$ & $\mathrm{~km}$ & $\mathrm{n}$ & $\%$ & $\mathrm{~km}$ & $\mathrm{n}$ & $\%$ & $\mathrm{~km}$ \\
\hline \multicolumn{22}{|l|}{ Sexo } \\
\hline Masculino & 2 & 66.7 & 3,813 & 2 & 40 & 1,328 & 4 & 25 & 1,519 & 4 & 50 & 1,95 & 20 & 50 & 0,864 & 11 & 26.8 & 0,714 & & & \\
\hline Feminino & 1 & 33.3 & 2,242 & 3 & 60 & 1,592 & 12 & 75 & 0,628 & 4 & 50 & 1,188 & 20 & 50 & 0,799 & 30 & 73.2 & 0,548 & 4 & 100 & 0,666 \\
\hline \multicolumn{22}{|l|}{ NA } \\
\hline \multicolumn{22}{|l|}{ Grau de instrução } \\
\hline Não alfabetizado & & & & & & & & & & & & & & & & 1 & 2.4 & 0,707 & & & \\
\hline Pré-escola & & & & & & & & & & & & & 2 & 5 & 0,848 & 9 & 22 & 0,658 & & & \\
\hline 10 grau incompleto & & & & 1 & 20 & 3,617 & 1 & 6.2 & 0,182 & 1 & 12.5 & 0,75 & 8 & 20 & 0,696 & 10 & 24.4 & 0,649 & & & \\
\hline 1o grau completo & & & & 3 & 60 & 1,01 & 5 & 31.2 & 0,576 & & & & 5 & 12.5 & 0,436 & 11 & 26.8 & 0,55 & & & \\
\hline 20 grau incompleto & 1 & 33.3 & 4,076 & 1 & 20 & 0,786 & 1 & 6.2 & 2,468 & 7 & 87.5 & 1,686 & 24 & 60 & 0,944 & 5 & 12.2 & 0,604 & 2 & 50 & 0,256 \\
\hline 2o grau completo & 2 & 66.7 & 2,896 & & & & 7 & 43.8 & 0,971 & & & & 1 & 2.5 & 1,741 & 4 & 9.8 & 0,382 & 2 & 50 & 1,067 \\
\hline Superior incompleto & & & & & & & & & & & & & & & & 1 & 2.4 & 0,578 & & & \\
\hline Superior completo & & & & & & & 2 & 12.5 & 0,642 & & & & & & & & & & & & \\
\hline \multicolumn{22}{|l|}{ Condição de renda } \\
\hline Tem renda & 3 & 100 & 3,289 & 4 & 80 & 1,39 & 14 & 87.5 & 0,786 & & & & 5 & 12.5 & 0,714 & 6 & 14.6 & 0,598 & & & \\
\hline Não tem renda & & & & & & & & & & 8 & 100 & 1,569 & 35 & 87.5 & 0,849 & 35 & 85.4 & 0,592 & 4 & 100 & 0,666 \\
\hline Não respondeu & & & & 1 & 20 & 1,87 & 2 & 12.5 & 1,304 & & & & & & & & & & & & \\
\hline \multicolumn{22}{|l|}{ Idade } \\
\hline Criança (até 10 anos) & & & & & & & & & & 1 & 12.5 & 0,75 & 5 & 12.5 & 1,317 & 17 & 41.5 & 0,633 & & & \\
\hline Adulto (10-60 anos) & 3 & 100 & 3,289 & 4 & 80 & 0,954 & 15 & 6.2 & 0,889 & 7 & 87.5 & 1,686 & 29 & 72.5 & 0,857 & 23 & 56.1 & 0,533 & 4 & 100 & 0,666 \\
\hline Idoso (mais 60 anos) & & & & 1 & 20 & 3,617 & 1 & 93.8 & 0,271 & & & & 6 & 15 & 0,67 & 1 & 2.4 & 1,263 & & & \\
\hline Idade média (anos) & & & 30.00 & & & 43.40 & & & 39.13 & & & 13.5 & & & 20.97 & & & 23.73 & & & 29.75 \\
\hline
\end{tabular}




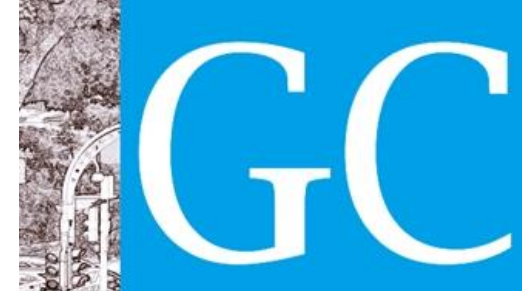

Revista Nacional de

Gerenciamento de Cidades

Tabela 4 - Variáveis sociodemográficas e distâncias por subcategorias (parte 2)

\begin{tabular}{|c|c|c|c|c|c|c|c|c|c|c|c|c|c|c|c|c|c|c|c|c|c|c|c|c|}
\hline & \multicolumn{6}{|c|}{ Compras } & \multicolumn{9}{|c|}{ Saúde } & \multicolumn{9}{|c|}{ Recreação } \\
\hline & \multicolumn{3}{|c|}{ Comércio geral } & \multicolumn{3}{|c|}{ Comércio de alim. } & \multicolumn{3}{|c|}{$\begin{array}{c}\text { Consultórios e } \\
\text { farmácias }\end{array}$} & \multicolumn{4}{|c|}{ UPA e hospitais } & \multicolumn{2}{|c|}{ UBS } & \multicolumn{3}{|c|}{ Igrejas } & \multicolumn{3}{|c|}{ Visitas } & \multicolumn{3}{|c|}{ Lazer } \\
\hline & $\mathrm{n}$ & $\%$ & $\mathrm{~km}$ & $\mathrm{n}$ & $\%$ & $\mathrm{~km}$ & $\mathrm{n}$ & $\%$ & $\mathrm{~km}$ & $\mathrm{n}$ & $\%$ & $\mathrm{~km}$ & $n$ & $\%$ & $\mathrm{~km}$ & $\mathrm{n}$ & $\%$ & $\mathrm{~km}$ & $\mathrm{n}$ & $\%$ & $\mathrm{~km}$ & $\mathrm{n}$ & $\%$ & $\mathrm{~km}$ \\
\hline \multicolumn{25}{|l|}{ Sexo } \\
\hline Masculino & & & & 3 & 27.3 & 0,932 & 1 & 25 & 0,432 & 2 & 33.3 & 0,581 & & & & 1 & 25 & 4,158 & 4 & 44.4 & 1,115 & 2 & 50 & 0,633 \\
\hline Feminino & 5 & 100 & 1,116 & 8 & 72.7 & 0,72 & 2 & 50 & 0,777 & 4 & 66.7 & 0,801 & 5 & 100 & 1,226 & 3 & 75 & 2,589 & 5 & 55.6 & 0,855 & 2 & 50 & 0,633 \\
\hline NA & & & & & & & 1 & 25 & 0,315 & & & & & & & & & & & & & & & \\
\hline \multicolumn{25}{|l|}{ Grau de instrução } \\
\hline Não alfabetizado & & & & 1 & 9.1 & 0,368 & 1 & 25 & 0,432 & 1 & 16.7 & 0,381 & & & & & & & & & & 1 & 25 & 0,437 \\
\hline Pré-escola & & & & & & & & & & 1 & 16.7 & 0,848 & & & & & & & 2 & 22.2 & 0,284 & 2 & 50 & 0,829 \\
\hline 10 grau incompleto & 1 & 20 & 0,483 & 2 & 18.2 & 0,991 & & & & 1 & 16.7 & 0,387 & 1 & 20 & 0,577 & & & & 2 & 22.2 & 1,283 & 1 & 25 & 0,437 \\
\hline 1o grau completo & & & & 4 & 16.4 & 0,371 & 1 & 25 & 0,777 & 3 & 50 & 0,916 & 3 & 60 & 1,573 & 1 & 25 & 4,145 & 3 & 33.3 & 1,582 & & & \\
\hline 20 grau incompleto & 1 & 20 & 0,107 & 1 & 9.1 & 0,368 & & & & & & & & & & & & & 2 & 22.2 & 0,427 & & & \\
\hline 2o grau completo & 3 & 60 & 1,663 & 1 & 9.1 & 1,869 & 1 & 25 & 0,264 & & & & 1 & 20 & 0,834 & 3 & 75 & 2,594 & & & & & & \\
\hline \multicolumn{25}{|l|}{ Superior incompleto } \\
\hline Superior completo & & & & 2 & 18.2 & 1,244 & 1 & 25 & 0,366 & & & & & & & & & & & & & & & \\
\hline \multicolumn{25}{|l|}{ Condição de renda } \\
\hline Tem renda & 2 & 40 & 0,298 & 8 & 72.7 & 0,903 & 4 & 100 & 0,46 & 4 & 66.7 & 0,708 & 3 & 60 & 1,029 & 3 & 75 & 2,594 & 4 & 44.4 & 1,255 & 4 & 100 & 0,633 \\
\hline Não tem renda & 3 & 60 & 1,661 & 3 & 27.3 & 0,446 & & & & 2 & 33.3 & 0,766 & 2 & 40 & 1,522 & 1 & 25 & 4,145 & 4 & 44.4 & 0,855 & & & \\
\hline Não respondeu & & & & & & & & & & & & & & & & & & & 1 & 11.1 & 0,293 & & & \\
\hline \multicolumn{25}{|l|}{ Idade } \\
\hline \multicolumn{25}{|l|}{ Criança (até 10 anos) } \\
\hline Adulto (10-60 anos) & 4 & 80 & 1,274 & 6 & 45.5 & 0,857 & 1 & 25 & 0,264 & 1 & 16.7 & 1,145 & 3 & 60 & 1,7 & 4 & 100 & 2,981 & 4 & 44.4 & 0,855 & & & \\
\hline Idoso (mais 60 anos) & 1 & 20 & 0,483 & 5 & 54.5 & 0,683 & 3 & 75 & 0,525 & 5 & 83.3 & 0,644 & 2 & 40 & 0,516 & & & & 5 & 55.6 & 1,063 & 4 & 100 & 0,633 \\
\hline Idade média (anos) & & & 33.80 & & & 61.00 & & & 50.75 & & & 64.17 & & & 51.80 & & & 44.50 & & & 61.67 & & & 78.50 \\
\hline
\end{tabular}

Fonte: Os autores, 2020 


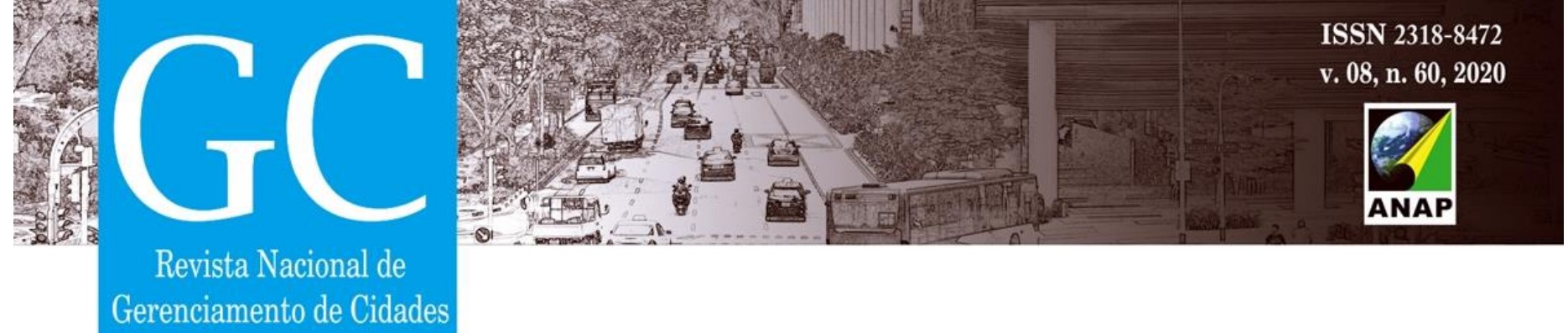

\section{CONCLUSÕES}

A redução de distâncias de destinos tem sido considerada para aumentar o deslocamento a pé. Neste estudo, observou-se uma prevalência de destinos de "Educação". Tal resultado dá subsídios para localização de equipamentos de educação. A concentração de maiores densidades de destinos nos deslocamentos a pé na Vila Oliveira, parece estar vinculado à discussão socioeconômica. Pode-se observar também que diferentes perfis sociodemográficos estão relacionados aos deslocamentos e às distâncias: idosos, geralmente, restritos ao ambiente do bairro; adultos podem percorrem maiores distâncias, em especial para Trabalho.

As limitações deste estudo foram decorrentes, principalmente, do banco de dados. A pequena amostra de viagens e os destinos reportados sem informações específicas diminuíram a amostragem.

O presente estudo evidencia a importância do entendimento dos estudos de caminhabilidade não apenas a partir da origem mas também do destino. Ainda a categorização dos destinos para a compreensão da caminhada utilitária, para definição de diferentes distâncias a partir de diferentes relações de sociodemográficas devem ser consideradas. Tais resultados contribuirão para subsidiar políticas públicas para a construção de cidades mais saudáveis e sustentáveis.

\section{AGRADECIMENTOS}

Ao Conselho Nacional de Desenvolvimento Científico e Tecnológico (CNPq) pela concessão da bolsa de Iniciação Científica, à CAPES pela bolsa de pós-graduação, à Fundação Araucária pela bolsa pós-doc e ao Grupo de pesquisa dos autores.

\section{REFERÊNCIAS BIBLIOGRÁFICAS}

BRASIL. Ministério do Trabalho e Emprego. Departamento de Emprego e Salário. RAIS 2014: Brasília, 2016. Disponível em: <http://acesso.mte.gov.br/rais/>. Acesso em 20 abr. 2020.

CAMPOLI, Julie. Made for Walking: Density and Neighborhood Form. 2. ed. Cambridge, Massachusetts: Lincoln Institute of Land Policy, 2012.

CERIN, Ester et al. Destinations that matter: Associations with walking for transport. Health \& Place, v. 13, n. 3, p. 713-724, 2007.

CERIN, Ester et al. Walking for transportation in Hong Kong Chinese urban elders: a cross-sectional study on what destinations matter and when. International Journal of Behavioral Nutrition and Physical Activity, v. 10, n. 1, p. 78, 2013.

CERVERO, Robert; KOCKELMAN, Kara. Travel demand and the 3Ds: Density, diversity, and design. Transportation Research Part D: Transport and Environment, v. 2, n. 3, p. 199-219, 1997.

CHATMAN, Daniel G. How Density and Mixed Uses at the Workplace Affect Personal Commercial Travel and Commute Mode Choice. Transportation Research Record: Journal of the Transportation Research Board, v. 1831, n. 1, p. 193201, 2003. 


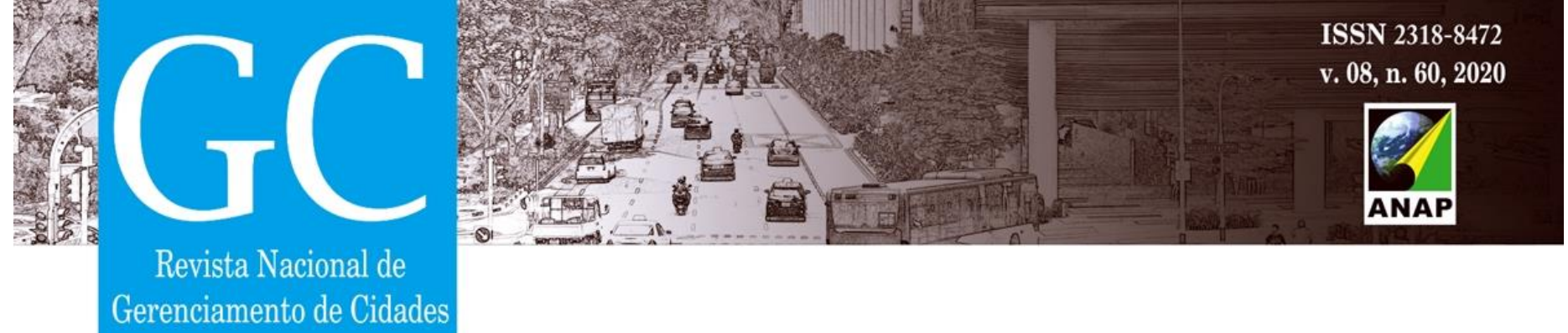

CHILLÓN, P. et al. Socio-economic factors and active commuting to school in urban Spanish adolescents: the AVENA study. The European Journal of Public Health, v. 19, n. 5, p. 470-476, 2009.

CHILLÓN, P. et al. A longitudinal study of the distance that young people walk to school. Health \& Place, v. 31, p. 133137, 2015.

CHODZKO-ZAJKO, Wojtek J. et al. Exercise and Physical Activity for Older Adults. Medicine \& Science in Sports \& Exercise, v. 41, n. 7, p. 1510-1530, 2009.

D'HAESE, Sara et al. Criterion distances and environmental correlates of active commuting to school in children. International Journal of Behavioral Nutrition and Physical Activity, v. 8, n. 1, p. 88, 2011.

DOESCHER, Mark P. et al. The built environment and utilitarian walking in small U.S. towns. Preventive Medicine, v. 69, p. 80-86, 2014.

FRANK, L. D. et al. The development of a walkability index: application to the Neighborhood Quality of Life Study. British Journal of Sports Medicine, v. 44, n. 13, p. 924-933, 2010.

FRANK, Lawrence et al. Urban Form Relationships with Walk Trip Frequency and Distance among Youth. American Journal of Health Promotion, v. 21, n. 4 suppl, p. 305-311, 2007.

GUO, Xiaoping; BLACK, John; DUNNE, Michael. Crossing pedestrians and dynamic severance on urban main roads. Road and Transport Research, v. 10, n. 3, p. 84-98, 2001.

HABIBIAN, Meeghat; HOSSEINZADEH, Aryan. Walkability index across trip purposes. Sustainable Cities and Society, v. 42 , p. $216-225,2018$

HALLAL, Pedro Curi et al. Prevalência de sedentarismo e fatores associados em adolescentes de 10-12 anos de idade. Cadernos de Saúde Pública, v. 22, n. 6, p. 1277-1287, 2006.

HOUSTON, D. Implicações do problema da unidade de área modificável na avaliação de correlatos do ambiente construído de atividade física moderada e vigorosa. Geografia Aplicada, v. 50, p. 40-47, 2014.

IBGE, Agência de Notícias. PNS 2013: três em cada quatro brasileiros costumam buscar atendimento médico na rede pública de saúde. Rio de Janeiro: IBGE, 2017. Disponível em: <agenciadenoticias.ibge.gov.br/agencia-sala-deimprensa/2013-agencia-de-noticias/releases/10138-pns-2013-tres-em-cada-quatro-brasileiros-costumam-buscaratendimento-medico-na-rede-publica-de-saude>. Acesso em 23 abr. 2020.

IBGE, Cidades. Rolândia. Rio de Janeiro: IBGE, 2020. Disponível em: <cidades.ibge.gov.br/brasil/pr/rolandia/panorama>. Acesso em 2 mai. 2020.

KANG, Bumjoon et al. Differences in behavior, time, location, and built environment between objectively measured utilitarian and recreational walking. Transportation Research Part D: Transport and Environment, v. 57, p. 185-194, 2017.

KENYON, Anna; PEARCE, Jamie. The socio-spatial distribution of walkable environments in urban scotland: A case study from Glasgow and Edinburgh. SSM - Population Health, v. 9, p. 100461, 2019.

KING, Tania Louise et al. Does the presence and mix of destinations influence walking and physical activity? International Journal of Behavioral Nutrition and Physical Activity, v. 12, n. 1, p. 115, 2015.

KOTLER, Phillip, ARMSTRONG, Gary. Princípios do Marketing. Tradução de Arlete Simille Marques e Sabrina Cairo. 9.ed. São Paulo. Prentice Hall, 2007.

LARA, Daniela Vanessa Rodriguez; RODRIGUES DA SILVA, Antônio Nélson. Equity issues associated with transport barriers in a Brazilian medium-sized city. Journal of Transport \& Health, v. 14, p. 100582, 2019. 
